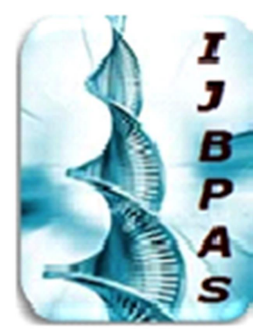

International Journal of Biology, Pharmacy and Allied Sciences (IJBPAS) 'A Bridge Betuen Caboratory and QRader'

WWW.iibpas.com

\title{
SCREENING AND ISOLATION OF Klebsiella Spp. FROM COPD PATIENTS FOR BIOCHEMICAL AND GENETIC ANALYSIS UNVEILING ITS PATHOGENIC RELEVANCE
}

\author{
SAFEENA KHANUM, TALLURI RAMESHWARI K R AND SUMANA K* \\ Department of Microbiology, Faculty of Life Sciences, JSS Academy of Higher Education \\ and Research, S S Nagar, Mysuru, Karnataka \\ *Corresponding Author: Dr. Sumana K: E Mail: sumana.k@jssuni.edu.in
}

Received $14^{\text {th }}$ Jan. 2021; Revised $13^{\text {th }}$ Feb. 2021; Accepted $12^{\text {th }}$ March 2021; Available online $1^{\text {st }}$ Nov. 2021 https://doi.org/10.31032/IJBPAS/2021/10.11.5703

\begin{abstract}
Chronic obstructive pulmonary disease (COPD) is the major cause of mortality rate all over the globe. An irreversible decline in lungs function leads to permanent alteration in surface area of alveoli. COPD decreases the immune of the patient leading to asthma, chronic bronchitis and other pulmonary diseases. The pathogenic organisms were isolated from the remnant sputum samples of COPD patients. Based on the phenotypic characteristics isolates suspected to be Klebsiella pneumonia were selected and tested for biochemical characterization using the pure colony. Up on performing Antibiotic susceptibility test using various antibiotics by standard Kirby Bauer method, all the isolated were found to be highly sensitive to chloramphenicol, than other antibiotics such as Meropenem, imipenem, ertapenem gentamycin, Co-trimoxazole, cefotaxime. To study the genetics and to identify the isolates at genetic level, molecular studies were performed through fingerprinting which makes easy identification. DNA was extracted and gel Electrophoresis technique was carried to check the quality of the DNA isolated. RAPD was performed at an appropriate annealing temperature. Upon performing gel electrophoresis the amplified products were observed, amongst which 4 samples appear similar which can be classified as a same subtype. And these obtained bands were compared which may aid to prepare a dendrogram of Klebsiella spp.
\end{abstract}


AIMS: The purpose of this study is to investigate, isolate the bacterial pathogens and study the microbiology of disease to increase our understanding about the pathogenesis and other consequences in COPD.

\section{Keywords: Chronic Obstructive Pulmonary Disease, Klebsiella pneumonia, antibiotic susceptibility test, RAPD}

\section{INTRODUCTION}

Developed countries are witnessing high morbidity and mortality owing to chronic obstructive pulmonary disease (COPD). The BOLD international study (Burden of Obstructive Lung Disease) estimated that about $10.1 \%$ was the prevalence of COPD for stage GOLD II or higher (Global Initiative for Chronic Obstructive Lung Disease), although it differed between countries [1]. For instance, Spain reported a prevalence of $10.2 \%$ patients between the age group 40 and 80 [2]. The disease progresses to severity due to acute exacerbation, which also indicates poor prognosis and a resulting high healthcare costs [3] [4]. Often, the COPD cases are underestimated because the report is not authenticated by the objective lung function tests [5]. A recent report suggests that about $4-10 \%$ of the Indian male population suffer from COPD as assessed by the spirometric method [6]. Considering its severity and the economic and healthcare burden caused by the pathogens causing COPD, it is becoming increasingly important for accurate and timely diagnostics of the causal agent.
Klebsiella pneumoniae is one of the chief human pathogen, first described by Carl Friedlander as a microbe isolated from the lungs of patients died from pneumonia [7]. It is a Gram-negative bacteria mostly associated with chronic obstructive pulmonary disease (COPD). They are rodshaped, catalase-positive, non-motile and utilize sugars such as glucose, xylose, malonate, Rhamnose for their carbon source. Upon fermentation, they show characteristic pink mucoid colonies on the MacConkey agar medium. The genome of $K$. pneumoniae differentiates strains into opportunistic, highly-virulent, multidrugresistant groups and delineates $K$. pneumoniae from the other cryptic species such as Klebsiella variicola and Klebsiella quasipneumoniae [8]. Klebsiella pneumoniae carbapenemases (KPCs) belongs to the class-A group with functional group b-lactamases $2 \mathrm{f}$ having serine at the active site and effectively splits carbapenems [9]. Capsule formation is one of the vital pathogenic factors of $K$. pneumoniae, and the type of capsules determines the degree of severity of the 
infection [10]. This capsule is the cause for many diseases such as bronchitis, emphysema and other respiratory conditions. Few strains of $K$. pneumoniae are capable of evading detection of the natural immune system by the host and are phagocytosis-resistant. Some of the factors that improve $K$. pneumoniae's survival and virulence include lipopolysaccharide (LPS), capsule polysaccharide (CPS), siderophores (e.g., aerobactin), and adhesion [11]. Currently, more than 13 Klebsiella pneumoniae carbapenemases (KPCs) subtypes have been identified in various regions worldwide. However, there is a lack of accurate diagnostic tool for the detection of the pathogen at the early stages of infection. Therefore, the aim of the present study was to compare the antibiotic susceptibilities, microscopic, biochemical and molecular typing of Klebsiella pneumonia isolates from patients with COPD

\section{OBJECTIVES}

$>$ Screening of the organisms - the sputum samples collected and tested for the presence of isolates

$>$ Biochemical tests - tests such as motility, MRVP, sugar utility test, and antibiotic susceptibility tests were done to characterize the isolates.
Comparative analysis of banding patterns in the isolated genomic DNA of the organism with the standard.

\section{MATERIALS AND METHODS}

\subsection{Collection and isolation}

Sputum samples were collected from victims newly hospitalized and before commencement of antibiotic treatment to them. All the samples collected were cultured on MacConkey Agar, Chocolate agar, and Blood agar at $37^{\circ} \mathrm{C}$ for $18-24$ hours. Later, isolates were sub cultured on to LB (Luria Bertani) broth and BHI (Brain Heart Infusion) broth medium.

\subsection{Microscopic examination by Gram's} staining technique

It is one of most versatile staining method in diagnostic microbiology laboratory. Gram's staining was performed and the pink colored colonies selected for further testing was subcultured into the LB broth and BHI broth. These broths are nutritionally rich and thus allow the growth of bacteria.

\subsection{Biochemical characterization}

Biochemical tests were done using pure cultures to identify and to differentiate the organism present in the sample. The biochemical assays viz., catalase, methyl red, citrate utilization test and TSI agar test for were performed for the isolated samples [12]. 


\subsection{Antibiotic Susceptibility Test}

This test was done by Kirby-Bauer disk diffusion method to determine the susceptibility of the organism towards various antibiotics. Known concentrations of the antibiotics were purchased from the supplier [Hi media - Cefotaxime SD0401PK, Co-Trimoxazole SD010-1PK. Chloramphenicol SD006-1PK. Methicillin SD019-1PK, Gentamicin SD016-1PK, Rifampicin SD030-1PK, and Tetracycline SD037-1PK].

Upon incubation, the zone of inhibition was measured using a caliper [17]. The zone of inhibition determines the sensitivity to the antibiotic namely, susceptible, intermediate or resistant.

\subsection{Molecular biological characterization}

\subsubsection{DNA extraction and quantification}

The DNA from the cultures grown on MacConkey agar medium was isolated as per Mora et al 1998 with minor modifications. The isolated DNA was checked on agarose gel electrophoresis and quantified using NanoDrop ND-1000 spectrophotometer V3.5 (NanoDrop Technologies, USA).

\subsubsection{Random Amplified Polymorphic DNA}

RAPD is a molecular typing method for differentiation bacterial samples and aids for producing a biochemical fingerprinting of the organism. In our study, RAPD typing was performed.

The oligonucleotide primers used for PCR amplification of specific segments is: Forward: 5' GTCCTTTGTCGATACTG 3', reverse 5' CAGGAAACAGCTATGAC 3'. The PCR was performed. The experimental set up was carried out in a Biorad T100 thermal cycler in a $50 \mu 1$ reaction volume comprising of $50 \mathrm{ng}$ DNA, $200 \mathrm{mM}$ each DNTPs, 2.5 U Red dye Taq (Merck Specialties Pvt. Ltd., Mumbai, India), 25 pMoles each of primers and 10X PCR buffer with $15 \mathrm{mM} \mathrm{MgCl2}$. The PCR was programmed as- $95^{\circ} \mathrm{C}-8 \mathrm{~min}$ followed by 35 cycles of $95^{\circ} \mathrm{C}-15 \mathrm{~s}$, annealing at $56^{\circ} \mathrm{C}$ $-30 \mathrm{~s}$ and an extension at $72^{\circ} \mathrm{C}-60 \mathrm{~s}$.

\section{RESULTS}

Screening and Isolation: The samples are screened for the microorganisms, through different selective media.

The sputum samples inoculated on to differential media such as MacConkey, blood agar, and chocolate agar media revealed a varied growth of colonies with different phenotypic appearances. As in Figure 1(a) the dark pink colonies are suspected to be Serratia spp, figure (b) and (c) shows the growth of mixed colonies which are creamy and pink colored. The pink colonies could be staphylococcus spp. In Figure (d) the pink mucoid colonies are seen, which is the phenotypic characteristic 
of Klebsiella pneumoniae. Klebsiella

pneumoniae is capsulated bacteria and therefore the colonies are sticky in appearance. The observations were recorded after 18 hours incubation period.

\section{Staining technique:}

Through phenotypic characteristics, the pink colored mucoid, sticky colonies were selected for Gram's staining which revealed that the organism is Gramnegative rods under microscope (Figure 2).

\section{Biochemical tests results}

In the present study, the microbial culture obtained from the sputum samples were subjected for biochemical assays. Klebsiella pneumonia cultures tested for Simmons citrate agar test revealed that the color of the media changed from green to bring blue suggesting the utilization of citrate for its carbon source. Further, in the TSI agar slant test the growth of culture led to the change in the color of the media indicating that there is no gas production and the organism ferments sugars such as Dextrose, Lactose/ sucrose (Figure 3).

\section{Antibiotic Susceptibility Test}

The isolates showed variation in the zone of inhibition for all the antibiotics at different concentration. Though the isolates appeared to similar but the effect of antibiotic was different. While some isolates showed resistance towards the antibiotics, some other showed susceptibility. Overall, the susceptibility ranged from 0-26 mm, where 0-12 suggests intermediately susceptible and 24-26 shows highly susceptible. Among all the antibiotics, Klebsiella pneumonia exhibited resistance to methicillin antibiotic (Table 1).

\section{Molecular biological characterization}

The 11 samples amplified are showing varied banding patterns. Among these sample no. 2, 8, 10 and 11 shows the similar banding patterns. These isolates from different sputum samples of COPD patients appear similar and can be classified as the same subtype. Using RAPD analysis, probe positive isolates from COPD patients were characterized from the sputum samples (Figure 4). 

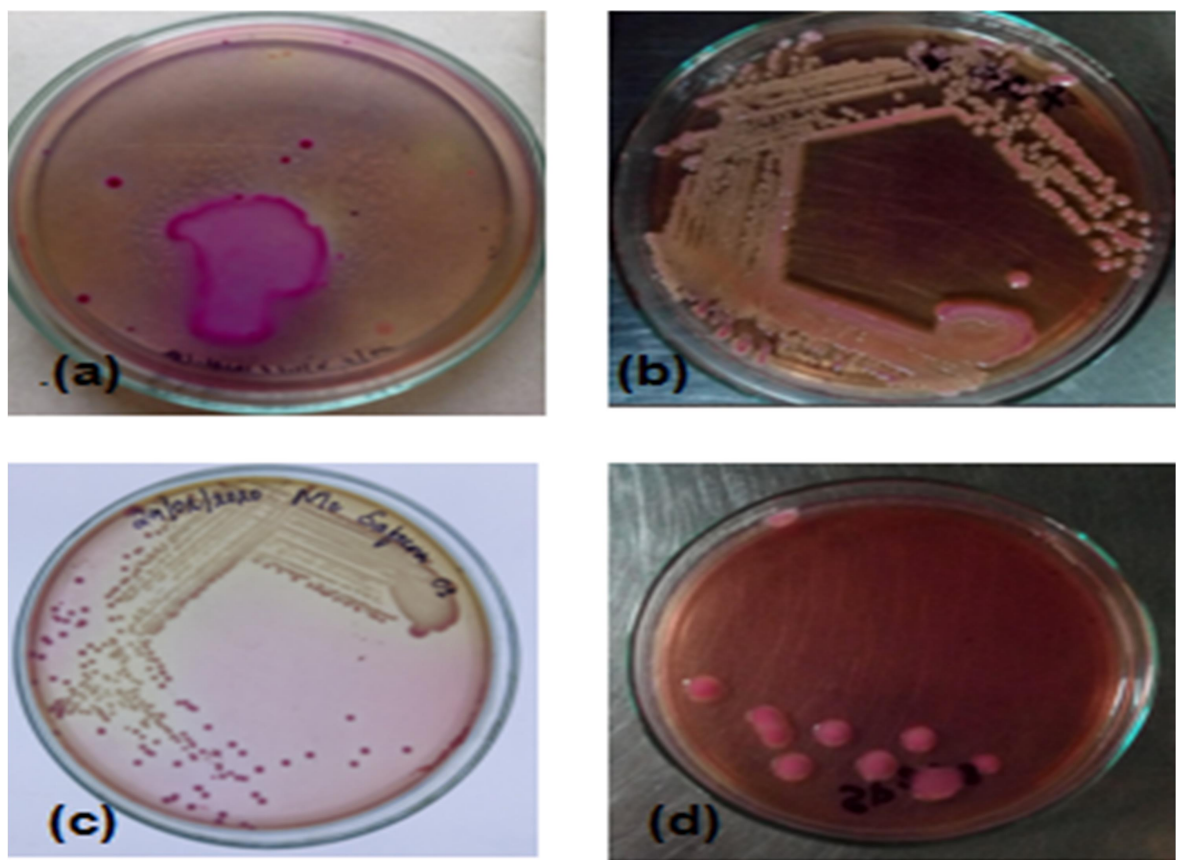

Figure 1 (a-d): The direct inoculation of sputum sample on MacConkey agar medium resulted in growth of varied colonies

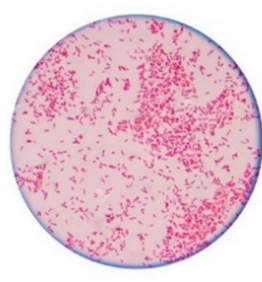

(a)

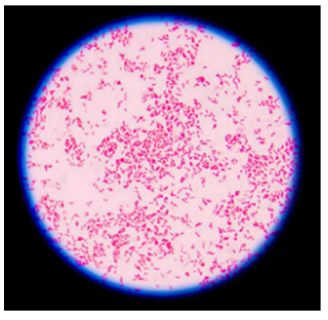

(b)

Figure 2: Gram's staining images

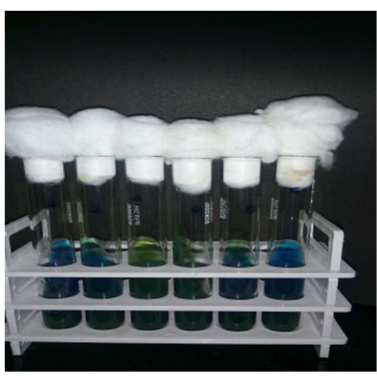

(a)

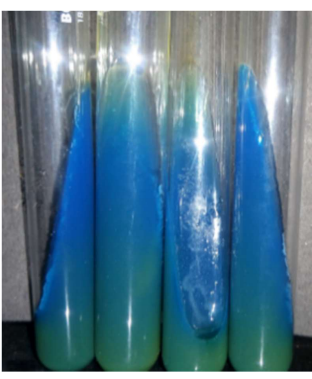

(b)

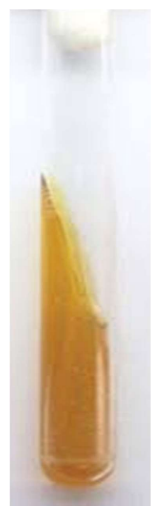

(c)

Figure 3: Biochemical tests (a \& b) Simmon citrate agar test, (c) TSI agar slant test 


\begin{tabular}{|c|c|c|c|}
\hline Antibiotics. & Resistant & Zone strength & Susceptible range $[\mathrm{mm}]$ \\
\hline Gentamicin [50mcg] & Nil & + & $9-12$ \\
\hline Chloramphenicol [30mcg] & Nil & +++ & 24-26 \\
\hline Rifampicin [15mcg] & Nil & + & $5-9$ \\
\hline Co-trimoxazole [25mcg] & Nil & + & $12-14$ \\
\hline Tetracycline [30mcg] & & + & $0-2$ \\
\hline Methicillin [5mcg] & Resistance & -- & -- \\
\hline Cefotaxime $[30 \mathrm{mcg}]$ & Nil & $+/-$ & $5-11$ \\
\hline Meropenem [10mcg] & Nil & + & 13-16 \\
\hline Ertapenem [10mcg] & Nil & + & 10-14 \\
\hline Imipenem [10mcg] & Nil & + & $12-16$ \\
\hline Tetracycline $1 \mathrm{mg}$ conc & Nil & + & $1-3$ \\
\hline
\end{tabular}

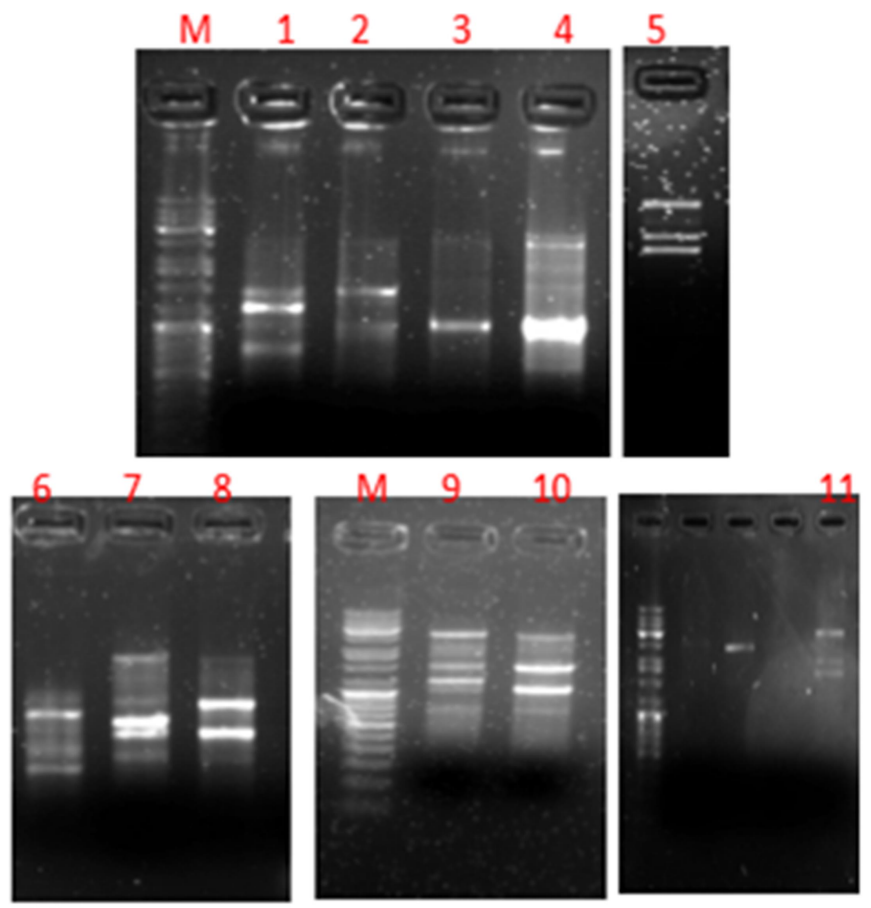

Figure 4: Results of RAPD analysis of isolates obtained from sputum samples of patients with COPD performed with primers M13

\section{DISCUSSION}

When the samples were inoculated on differential media (MacConkey agar), through phenotypical identification, the pink colored mucoid colonies were selected for further testing. Klebsiella spp generally exhibit convex mucoidal colonies that have a sticky appearance. Few samples collected after giving the first dose of the antibiotics showed growth of Klebsiella spp and other bacteria's such as Serratia and staphylococcus species. These bacteria also grow as pink colored colonies on MacConkey agar media. The biochemical tests such as catalase, methyl red Voges Proskauer, Simmon citrate agar, triple slant iron agar tests were carried out and the results were similar to that of Klebsiella pneumoniae strains. The similar 
identification was performed by Osman et al [19].

Antibiotic susceptibility testing using the Kirby Bauer disk diffusion method showed that the organism showed a variation in sensitivity pattern. All the isolates tested were sensitive to chloramphenicol with a large zone of inhibition. Chloramphenicol is a static drug that inhibits the protein synthesis in bacterial cell by preventing the elongation of the protein chain and inhibiting the peptidyl transferase activity of the bacterial ribosomes. It binds to the residues of 23SRNA of the 50S ribosomes. The isolates were also sensitive to Meropenem, imipenem, ertapenem, gentamycin, Co-trimoxazole, cefotaxime and resistant to Tetracycline, methicillin, with greater extent. Similar work was done by Makled et al [20]. Aim of their study was to study the incidence of aerobic bacteria in COPD patients, and they found that Klebsiella pneumonia was the most predominant. They carried out antibiotic susceptibility testing and showed the rate of resistance acquired by the organism to certain antibiotics.

The RAPD banding pattern of Klebsiella pneumoniae strains obtained from 11 different amplification is similar to that reported in the previous studies, which showed genetic similarity between Klebsiella pneumoniae and Klebsiella oxytoca strains $[\mathbf{2 1}, \mathbf{2 2}]$. Thebanding pattern of isolates in figure are similar to the pattern obtained by the other studies. Hence it is concluded that these isolates are K. pneumoniae.

The antibiotic resistance is increasing rapidly. ESBL cases are profoundly predominant among Klebsiella spp. ESBL producers are sensitive to carbapenems. Increase in Carbapenemases producing Klebsiella spp are creating the treatment problem complicated with morbidity, mortality as well as great economic loss [22]. Further, by knowing the sequence data of these isolates, a common primer of 18 to 22 bases could be designed, benefitting for early and rapid detection of the pathogen.

\section{SUMMARY AND CONCLUSION}

The present study aimed at characterization of Klebsiella pneumonia samples from the sputum of COPD patients. In this study, the microscopic and biochemical characterization was supported by the molecular characterization of the pathogen using RAPD method. Based on the RAPD markers, species specific primers can be designed that can assist in rapid and early detection of this pathogen. This research study can be optimized by creating a marker for the identification of highly resistant strains of Klebsiella pneumonia which also gives space for the study of mutants. 


\section{ACKNOWLEDGEMENT}

The authors are thankful to Division of Microbiology \& Tissue Culture, Department of Water and Health, Faculty of Life Sciences, JSS Academy of Higher Education \& Research, Mysuru.

\section{REFERENCES}

[1] Buist AS, McBurnie MA, Vollmer WM, et al. International variation in the prevalence of COPD (the BOLD Study): a population-based prevalence study, Lancet, 2007, vol. 370 (pg. 74150)

[2] Miravitlles M, Soriano JB, GarciaRio F, et al. 2009. Prevalence of COPD in Spain: impact of undiagnosed COPD on quality of life and daily life activities, Thorax, vol. 64 (pg. 863-868)

[3] Soler-Cataluña JJ, Martinez-Garcia MA, Roman SP, et al. 2005. Severe acute exacerbations and mortality in patients with chronic obstructive pulmonary disease. Thorax, vol. 60 (pg. 925-31)

[4] Sethi S, Murphy TF. Infection in the pathogenesis and course of chronic obstructive pulmonary disease, N Engl J Med, 2008, vol. 359 (pg. 2355-65)

[5] Evans J, Chen Y, Camp PG, Bowie DM, McRae L. 2014. Estimating the prevalence of COPD in
Canada: Reported diagnosis versus measured airflow obstruction. Health Rep. 2014 Mar; 25(3):3-11.

[6] Vimala, L. R., Gibikote, S., Christopher, D. J., Irodi, A., \& Rajan, M. (2018). Correlation of Digital Chest Radiograph Parameters with Pulmonary Function Test and BODE Index for Diagnosis and Prognostication in Indian COPD Patients. J Lung PulmRespir Res, 5(1), 00154.

[7] Friedlaender, C. Archiv Patholog Ueber die Schizomycetenbei der acutenfibrösen Pneumonie. Anat. Physiol. Klinische Med.1882; 87, 319-324.

doi: 10.1007/BF01880516.

[8] Martin RM and Bachman MA. Colonization, Infection, and the Accessory Genome of Klebsiella pneumoniae. (2018) Front. Cell. Infect. Microbiol. 8:4. doi: 10.3389/fcimb.2018.00004

[9] Michelle K. Paczosa, JoanMecsas. Klebsiella pneumoniae: Going on the Offense with a Strong Defense: 2016 Microbiol. Mol. Biol. Rev. 80: 629-661. doi:10.1128/MMBR.00078-15.

[10] Queenan AM, Bush K. Carbapenemases: the versatile blactamases. Clin. Microbiol Rev 2007; 20: 440e58 
[11] Pan Y-J, Lin T-L, Chen Y-H, et al. Capsular Types of Klebsiella pneumoniae Revisited by wzc Sequencing. PLoS ONE 2013; 8(12): e80670. doi:10.1371/journal.pone.0080670

[12] Dimri, A. G., Chaudhary, S., Singh, D., Chauhan, A., \& Aggarwal, M. L. (2020) Morphological and biochemical characterization of food borne Gram-positive and Gramnegative bacteria. Science Archives Vol. 1 (1), 16-23

[13] Brisse S, Grimont F, Grimont PAD. The Genus Klebsiella. New York, NY, USA: Springer Science+Business Media, LLC; 2006. p. 159-96.

[14] Kirby, W. M. M., G. M. Yoshihara, K. S. Sundsted, et al. Clinical usefulness of a single disc method for antibiotic sensitivity testing. Antibiotics Annu. 1956-1957:892.

[15] Jorgensen, J. H., and J. D. Turnidge. Susceptibility test methods: dilution and disk diffusion methods, 2007. p. 1152-1172. In P. R. Murray, E. J. Baron, J. H. Jorgensen, M. L. Landry, and M. A. Pfaller (ed.), Manual of clinical microbiology, $9^{\text {th }}$ ed. ASM Press, Washington,

D.C.

[16] Winn, Jr., W., et al. Konemann's color atlas and diagnostic text of microbiology, 6th ed., 2006. p. 945-1021. Lippencott Williams \& Wilkins Publishers, Philadelphia, PA.

[17] Kirby-Bauer Disk Diffusion Susceptibility Test Protocol. Jan Hudzicki American society of microbiology 2009.

[18] NCCLS. Performance standards for antimicrobial disc susceptibility tests. Approved standard NCCLS publication M2-A5, Villanova, PA,USA, 1993

[19] Osman et al. Comparing conventional, biochemical and genotypic methods for accurate identification of Klebsiella pneumoniae in Sudan. Access Microbiology 2020; 2 DOI 10.1099/acmi.0.000096

[20] Makled et al. Klebsiella pneumonia and chronic obstructive pulmonary disease, April 2019 Volume 28 / No. 2; 51-60

[21] M. V. Pimenta Rodrigues et al. Assessment of Genetic Relationship between Klebsiella pneumoniae and Klebsiella 
oxytoca samples isolated from a dental offices. J. Venom. Anim.

Toxins incl. Trop. Dis., 2008, 14, 4, p. 704

[22] Subedi et al. Antibiotic Susceptibility Test of Klebsiella pneumoniae and $K$. oxytoca Isolated from Different Clinical Samples and Perform Random Amplified Polymorphic DNA among $K$. pneumoniae. BMRJ, 2016; 12(6): 1-11, Article no. BMRJ.23376.

DOI:10.9734/BMRJ/2016/23376 